\title{
Anesthetic Management for Caesarean Section in a Patient with Impetigo Herpetiformis
}

\author{
Nan-Seol Kim \\ Department of Anesthesiology and Pain Medicine, Soonchunhyang University Cheonan Hospital, Soonchunhyang University College of Medicine, Cheonan, \\ Korea
}

Impetigo herpetiformis (IH) is an extremely rare pustular disorder and potentially life-threatening condition for both mother and fetus. Intrauterine growth retardation, fetal abnormalities, and even fetal/neonatal death can occur with worsening maternal disease and are probably related to placental insufficiency. Maternal risk is linked to fluid and electrolyte abnormalities, in particular, hypocalcemia-induced convulsions and sepsis. Therefore, early recognition is crucial to reduce both maternal and fetal morbidities, and a patient with $\mathrm{IH}$ may require emergency caesarean delivery. Here, we report a case of a 34-year-old pregnant woman with $\mathrm{IH}$ who underwent successful urgent general anesthesia for caesarean section.

Keywords: Anesthesia; Impetigo; Pregnancy; Psoriasis

\section{INTRODUCTION}

Impetigo herpetiformis (IH), also called generalized pustular psoriasis (GPP) of pregnancy, is a very rare pustular disorder of unknown etiology. It is characterized by a severe, generalized pustular skin eruption. Although there have been reports of disease emergence during the first trimester as well as the postpartum period, it typically occurs during the third trimester of pregnancy [1].

$\mathrm{IH}$ has been known to resolve spontaneously after delivery, but it can lead to a potentially life-threatening condition for the fetus and mother. Intrauterine growth retardation, fetal abnormalities, and even fetal/neonatal death can occur with worsening maternal disease and are probably related to placental insufficiency [2]. Maternal risk is linked to fluid and electrolyte abnormalities, in particular, hypocalcemia-induced convulsions and sepsis [3]. Therefore, early recognition is crucial to reduce both maternal and fetal morbidities, and a patient with IH may require emergency caesarean delivery.

Here, we report a case of a pregnant woman with IH who un- derwent successful urgent general anesthesia for caesarean section. After delivery, IH did not resolve with systemic corticosteroid and required administration of acitretin.

\section{CASE REPORT}

A 34-year-old multiparous woman (gravida 2, para 1) was referred to Soonchunhyang University Cheonan Hospital at 32 weeks gestation with diffuse, painful, and pruritic desquamative macular lesions. The macular lesions first presented on the nipple area 4 weeks prior to referral and subsequently spread to cover much of her abdomen, back, and groin (Fig. 1). Some lesions were also present on the patient's upper and lower extremities (Fig. 2). When the symptoms started, she thought it was simple psoriasis and did not seek medical advice during the first week. She initially presented to the local dermatology clinic and was treated for psoriasis for 2 weeks, but the symptoms were not controlled. IH was first diagnosed at the dermatology clinic, after which she was referred to our clinic.
Correspondence to: Nan-Seol Kim

Department of Anesthesiology and Pain Medicine, Soonchunhyang University Cheonan Hospital,

Soonchunhyang University College of Medicine, 31 Suncheonhyang 6-gil, Dongnam-gu, Cheonan 31151, Korea

Tel: +82-41-570-2722, Fax: +82-41-573-3559, E-mail: nskim1977@schmc.ac.kr

Received: Dec. 23, 2015/ Accepted after revision: Feb. 3, 2016
(C) 2016 Soonchunhyang Medical Research Institute This is an Open Access article distributed under the terms of the Creative Commons Attribution Non-Commercial License (http://creativecommons.org/licenses/by-nc/4.0/). 


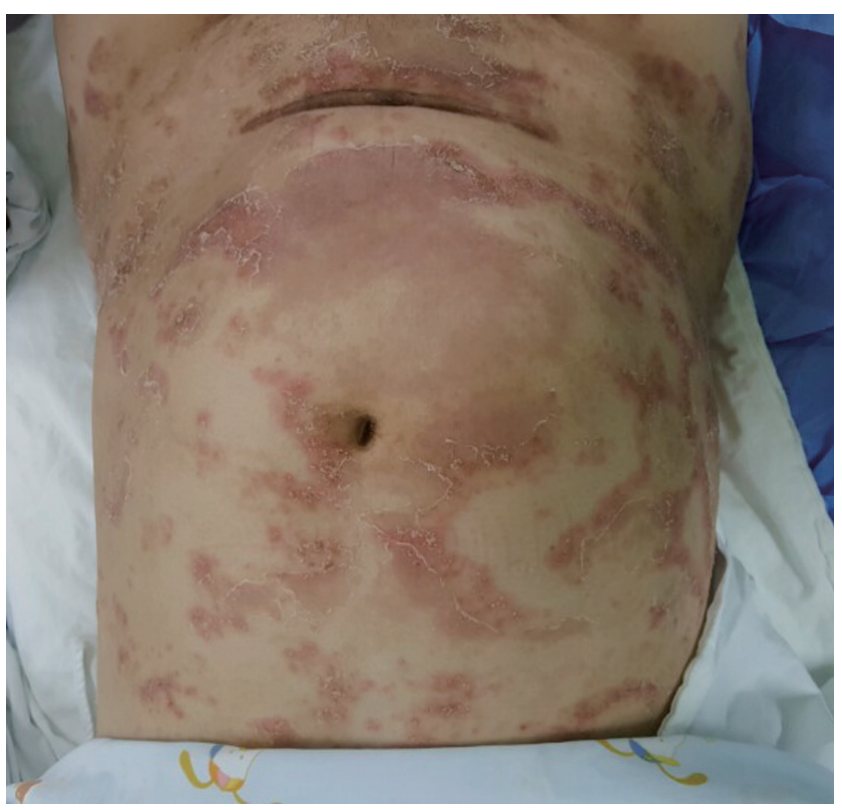

Fig. 1. Skin lesion of impetigo herpetiformis. Scaly erythematous patches on the abdomen.

The patient had a history of psoriasis, which was diagnosed at the age of 10, but she was not taking any medication for it. Her family had no past medical history of psoriasis. She had experienced an uncomplicated pregnancy and delivered a healthy baby. Laboratory evaluation revealed iron deficiency anemia (hemoglobin $10.0 \mathrm{~g} / \mathrm{dL}$ ) and elevated C-reactive protein (15.97 mg/L). Her white blood cell count was normal but had slight neutrophilia (85.9\%). Other biochemical levels, including albumin, calcium, and vitamin $\mathrm{D}$, were within the normal range. She had arthralgia and malaise but had stable vital signs except for tachycardia (heart rate 106 beats/min). A clinical diagnosis of $\mathrm{IH}$ was made and later confirmed by a pathologic examination.

Following admission, the patient was reviewed by a multidisciplinary team consisting of a dermatologist, an obstetrician, a neonatologist, and an anesthesiologist. Monitoring revealed a normal fetal heart rate. Obstetric ultrasonography also revealed adequate fetal growth (approximately 1,900 g) without any abnormal findings.

To avoid the risk of stillbirth and further complications to the mother, the decision was made to perform an urgent caesarean section. An obstetric anesthesiologist was consulted to give a preoperative evaluation prior to the urgent elective caesarean delivery. Fortunately, examination of the airway was normal, and given the severity of the lesions on her back, the need for general anesthesia was discussed with the patient. Once in the operating room, monitors were attached to the skin areas without lesions.

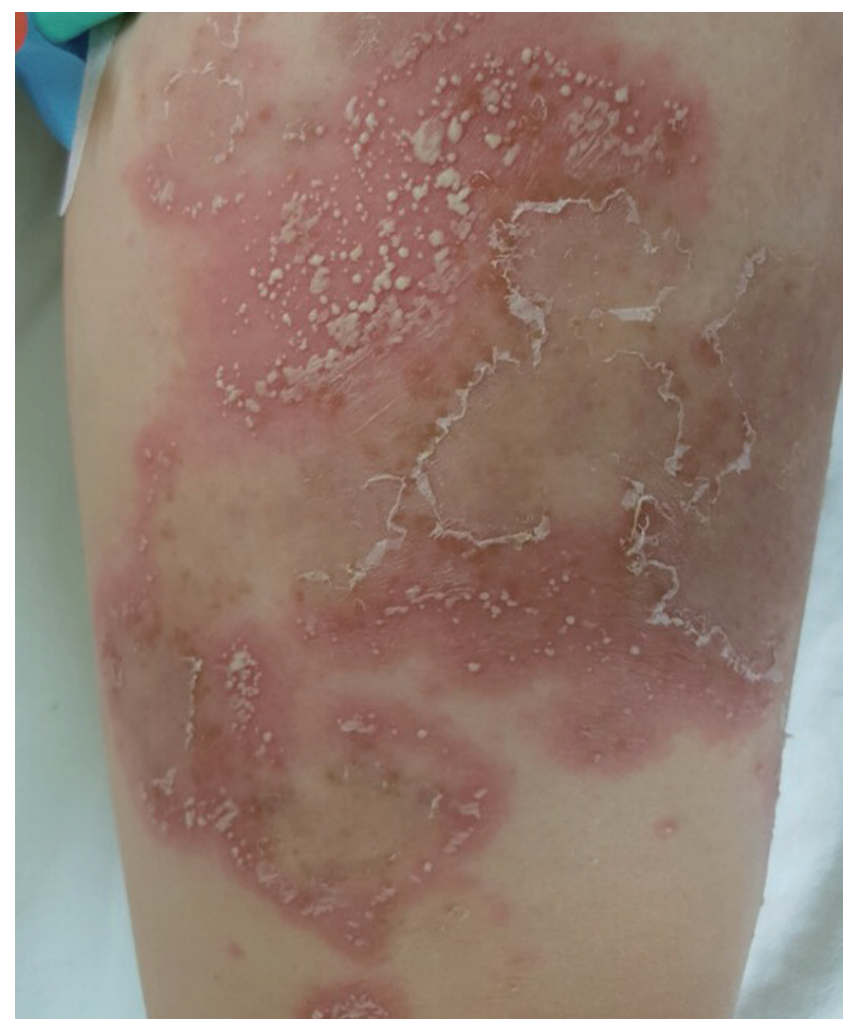

Fig. 2. Multiple tiny pustular skin lesions on the erythematous base on the leg.

After anesthetic induction with thiopental sodium and succinylcholine, tracheal intubation was performed in rapid sequence. The laryngoscopy view was documented as grade II. Maintenance of anesthesia was uneventful during the operation, and the trachea was extubated in the operating room. The neonatal intensive care team was present for resuscitation of the infant. Apgar scores were 6 and 7 at 1 and 5 minutes, respectively, and the birth weight was $1,780 \mathrm{~g}$. The neonate was initially apneic with little spontaneous movement and ultimately required tracheal intubation in the operating room due to poor respiratory effort. The baby was transferred to the neonatal intensive care unit.

After delivery, the patient was treated systemically with intravenous prednisone, which was switched to oral prednisone on day 2 . Because of pruritus, chlorpheniramine was added and antibiotics were also administered to prevent secondary infection. The treatment resulted in a transient partial response, but the disease was exacerbated after a few days. Even though the skin lesion persisted, the patient was discharged, receiving prednisone for 4 days postoperatively. At follow-up 8 days after delivery, no marked improvement was noted. Therefore, oral acitretin was initiated at 60 mg per day. Fortunately, there was partial improvement, and the 
dose of acitretin was reduced gradually during the postpartum period. Approximately 1 month after the cesarean section, the cutaneous symptoms disappeared leaving residual pigmentation. The baby was extubated 7 days after delivery and moved to general ward 20 days after delivery without complications.

\section{DISCUSSION}

IH was first described by Von Hebra in 1872 in five pregnant women with variants of generalized pustular lesions, characterized by an acute pustular eruption, which sadly resulted in the death of the fetuses and only one mother surviving [4]. The autopsies of the four women who died with variants of generalized pustular lesions did not reveal a definitive cause of death [4]. Triggering factors have been suggested to be hypocalcemia, hypoparathyroidism, infections, medications, climate/season, pregnancy, and steroid tapering [5,6], but due to the low number of cases, none of these have been confirmed. In our case, we considered that IH was a flare up of psoriasis triggered by pregnancy and related to hormonal effects.

There is a significant amount of controversy over whether $\mathrm{IH}$ is a variant of GPP or a separate disease entity. However, recently, IH has been considered as a variant of pustular psoriasis caused by hormonal changes during pregnancy $[1,7]$.

Early recognition of $\mathrm{IH}$ is of utmost importance as maternal and fetal well-being may be compromised due to misdiagnosis and inappropriate treatment. Despite the risk of morbidity and mortality to the mother and the fetus, our patient did not receive the appropriate treatment for several weeks due to misdiagnosis.

The diagnosis of $\mathrm{IH}$ is based mainly on clinical assessment of the presence of erythematous plaques, secondarily bordered by sterile pustules, which first develop in the skin folds and intertriginous areas and then extend centrifugally but may occur everywhere. Mucous membranes, including the tongue, mouth, and esophagus, are rarely involved. Subungual pustules may result in onycholysis $[1,3]$.

Patients with IH often have systemic signs and symptoms, including malaise, fever, nausea, vomiting, diarrhea, chills, and arthralgia. Laboratory findings are anemia, leukocytosis, an elevated erythrocyte sedimentation rate/C-reactive protein level and hypoalbuminemia, secondary hypocalcemia, or low serum levels of vitamin D [8]. Cardiac and renal failure may also occur [3].

From an anesthetic point of view, the main concern is whether there is involvement of the head and neck, especially airway edema for airway management. Furthermore, if lesions exist on the back, whether it is possible to perform regional anesthesia is of concern. Although IH is not a contraindication to spinal anesthesia, areas of chronically inflamed or diseased skin are best avoided as sites of administration [2]. In our case, active lesions over her lumbar region precluded regional anesthesia. Fortunately, there was no involvement of the head or neck and therefore no difficulty in performing intubation for general anesthesia. Also, there were no problems in monitoring. However, if the potential for airway edema exists, a difficult intubation should be expected. Also, if there are widespread pustular skin lesions in the monitoring areas, extreme care is required during electrocardiogram and blood pressure monitoring, similar to that needed for a patient with severe burns. In addition, if onycholysis is present due to nail bed involvement, the pulse oximeter should be placed on the ear.

A standard treatment guideline for IH has not been established, but cyclosporine, infliximab, oral prednisone, and topical agents are proven useful first-line therapies [7]. Systemic antimicrobials must be used if the skin lesions are secondarily infected.

However, in severe cases, termination of pregnancy will be required followed by treatment with oral retinoids [1]. A major concern with the use of systemic retinoids is their high teratogenic potential; therefore, they should not be used in any patient who is pregnant or likely to become pregnant [7]. However, our patient did not respond well to systemic corticosteroid therapy, and she already had two children and did not want to have more. Therefore, the dermatologist decided to use acitretin as a treatment, obtaining satisfactory results.

In some cases in which the maternal and fetal conditions are not severe, the pregnancy will continue to term with treatment $[9,10]$; however, despite treatment, maternal and/or fetal deaths have been reported $[5,6]$. In our case, although the fetus and the mother were stable from a gynecological standpoint, urgent surgery was performed. It is uncertain what the best choice is considering the baby had to remain in the hospital for a month for treatment due to being premature.

In conclusion, $\mathrm{IH}$ is an extremely rare pustular disorder that requires early recognition and prompt treatment due to the increased perinatal risk of mortality. Therefore, dermatologists, obstetricians, neonatologist, and anesthesiologists must work together to improve the quality of life of the mother and contribute to a favorable outcome for the fetus. 


\section{REFERENCES}

1. Roth MM. Pregnancy dermatoses: diagnosis, management, and controversies. Am J Clin Dermatol 2011;12:25-41.

2. Samieh-Tucker A, Rupasinghe M. Anaesthesia for caesarean section in a patient with acute generalised pustular psoriasis. Int J Obstet Anesth 2007;16:375-8.

3. Oumeish OY, Parish JL. Impetigo herpetiformis. Clin Dermatol 2006;24: 101-4.

4. Hebra FV. On some affections of the skin occurring in pregnant and puerperal women. Am J Syphilog Dermatol 1873;4:156-9.

5. Jin H, Cho HH, Kim WJ, Mun JH, Song M, Kim HS, et al. Clinical features and course of generalized pustular psoriasis in Korea. J Dermatol 2015;42:674-8
6. Kondo RN, Araujo FM, Pereira AM, Lopes VC, Martins LM. Pustular psoriasis of pregnancy (impetigo herpetiformis): case report. An Bras Dermatol 2013;88(6 Suppl 1):186-9.

7. Robinson A, van Voorhees AS, Hsu S, Korman NJ, Lebwohl MG, Bebo BF Jr, et al. Treatment of pustular psoriasis: from the Medical Board of the National Psoriasis Foundation. J Am Acad Dermatol 2012;67:279-88.

8. Ulubay M, Keskin U, Fidan U, Cicek AF, Caliskan E, Karaca RE, et al. Case report of a rare dermatosis in pregnancy: impetigo herpetiformis. J Obstet Gynaecol Res 2015;41:301-3.

9. Imai N, Watanabe R, Fujiwara H, Ito M, Nakamura A. Successful treatment of impetigo herpetiformis with oral cyclosporine during pregnancy. Arch Dermatol 2002;138:128-9.

10. Shaw CJ, Wu P, Sriemevan A. First trimester impetigo herpetiformis in multiparous female successfully treated with oral cyclosporine. BMJ Case Rep 2011;2011:bcr0220113915. 